\title{
Peak External Intensity Decreases across Quarters during Basketball Games
}

\author{
Jordan L. Fox ${ }^{1,2}$, Hugo Salazar ${ }^{3}$, Franc Garcia ${ }^{4}$, Aaron T. Scanlan ${ }^{1,2}$
}

Affiliations: 'Central Queensland University, School of Health, Medical, and Applied Sciences, Rockhampton, QLD, Australia, ${ }^{2}$ Central Queensland University, Human Exercise and Training Laboratory, Rockhampton, QLD, Australia, ${ }^{3}$ University of the Basque Country, Faculty of Physical Activity and Sport Sciences, Vitoria-Gasteiz, Spain, ${ }^{4}$ Futbol Club Barcelona, Sport Performance Area, Barcelona, Spain

Correspondence: J. L. Fox, Central Queensland University, Building 81/1.16, Bruce Highway, Rockhampton, QLD 4702, Australia. Email: j.fox2@cqu.edu.au

\begin{abstract}
The purpose of this study was to compare peak external intensities across game quarters in basketball. Eight semi-professional male players were monitored using accelerometers. For all quarters, peak intensities were determined via moving averages for PlayerLoad/minute (PL·min-1) using sample durations of $15 \mathrm{~s}, 30 \mathrm{~s}, 1 \mathrm{~min}, 2$ min, $3 \mathrm{~min}, 4 \mathrm{~min}$, and $5 \mathrm{~min}$. Linear mixed models and effect sizes (ES) were used to compare peak intensities between quarters for each sample duration. Small decreases in peak PL.min-1 occurred between Quarters 1 and 4 for all sample durations ( $E S=0.21-0.49$ ). Small decreases in peak PL·min-1 were apparent between quarters 1 and 2 for 30-s, 1-min, and 3-min sample durations ( $E S=0.24-0.33$ ), and between quarters 3 and 4 for 2-5-min sample durations $(E S=0.20-0.24)$. Peak intensities decline across quarters with game progression in basketball, providing useful insight for practitioners to develop game-specific training and tactical strategies.
\end{abstract}

Keywords: accelerometer, microsensor, training prescription, worst-case scenario

@MJSSMontenegro

PEAK INTENSITY DURING BASKETBALL GAME QUARTERS

http://mjssm.me/?sekcija=article\&artid=209

Cite this article: Fox, J. L., Salazar, H., Garcia, F., \& Scanlan, A. T. (2021). Peak External Intensity Decreases across Quarters during Basketball Games. Montenegrin Journal of Sports Science and Medicine, 10 (1), 25-29. doi: $10.26773 /$ mjssm. 210304

\section{Introduction}

In basketball, players are exposed to intense physical demands during games. Specifically, games involve frequent multi-directional movements (Taylor et al., 2017) along with substantial running demands (Stojanović et al., 2018). Given the physically demanding nature of basketball, optimal preparation leading into games is of critical importance to ensure that players can withstand the demands faced, consequently increasing the likelihood of successful performance (Fox et al., 2019).

Quantifying player demands across the entire game as well as during game quarters (Garcia et al., 2020) is essential to provide reference workloads and identify performance deficits across games, which in turn can be used to inform training prescription. In considering the demands encountered by players, data are typically expressed as either external demands or internal responses. Specifically, external demands represent the training or game stimuli imposed on players, while internal responses relate to the psychological and physiological reactions of players to the imposed demands (Impellizzeri et al., 2019). With respect to training prescription, the external demands represent the activity dosage directly prescribed and

Received: 25 June 2020 | Accepted after revision: 9 July 2020 | First published online: 1 March 2021

(c) 2021 by the author(s). License MSA, Podgorica, Montenegro. This article is an open access article distributed under the terms and conditions of the Creative Commons Attribution (CC BY).

Conflict of interest: None declared. 
controlled by practitioners to bring about the desired responses and subsequent adaptations from players (Fox et al., 2019). In turn, it is essential to quantify the external demands experienced during games for training demands to prepare players for competitive scenarios effectively. In professional basketball players, external demands (total distance and player load) have been shown decrease (effect size $(\mathrm{ES})=1.27-1.31$, large) between Quarter 1 and 4 (Garcia et al., 2020). In addition, external demands (high-intensity activity and PlayerLoad, respectively) have been shown to decrease across Quarter 3 and 4 (ES = 1.4-3.2, large-very large) (Scanlan et al., 2015), as well as overtime periods ( $\mathrm{ES}=1.46$, large) (Scanlan et al., 2019), compared Quarters 1 and 2 during games in semi-professional players. Consequently, existing data suggest external demands decrease across games, likely as a function of changes in tactical approaches, and accumulated fatigue.

While understanding differences in external demands between game quarters is essential to prescribe training for basketball players more precisely, previous work has quantified total external load or average intensity across each quarter (Garcia et al., 2020; Scanlan et al., 2019). However, in better understanding the game demands experienced by players, the quantification of peak external intensities may provide further insights by determining the most demanding passages of game-play, also referred in the existing literature as "worstcase scenarios" (Cunningham et al., 2018). Specifically, understanding fluctuations in peak intensities between quarters may indicate player ability to sustain high-intensity activity across games for greater precision in prescribing training and managing fatigue-related outcomes. It is currently not clear whether trends reported in external demands across quarters are also apparent for metrics representing the most demanding passages of games. To date, no research has compared peak external intensities across game quarters in basketball, with only peak external intensities captured during entire games previously examined (Fox et al., 2020; Salazar \& Castellano, 2019). Therefore, the purpose of this study was to compare peak external intensities encountered by players across game quarters in basketball.

\section{Methods}

Eight semi-professional, male basketball players (age: $23 \pm$ 4 yr; stature: $191 \pm 8 \mathrm{~cm}$; body mass: $87 \pm 16 \mathrm{~kg}$; semi-professional playing experience: $5 \pm 2 \mathrm{yr}$ ) volunteered to participate in the study. All players belonged to the same team competing in the Queensland Basketball League, a second-tier, state-level Australian basketball team. Other players from the same team received limited playing time across the season $(<4$ min per game) and therefore were not included in the study. Prior to study commencement, players were screened for injuries or health conditions that may have prevented safe participation. All players were informed of the purpose of the study and any potential risks or benefits of participation before providing voluntary written informed consent prior to participating. All procedures were approved by an institutional Human Research Ethics Committee.

Across the season, 18 games were scheduled, with 1-3 games held each week between Friday and Sunday. Each game consisted of $4 \times 10$-min quarters, with 2 - and 15-min breaks between quarters and halves, respectively. Prior to study commencement, anthropometric data were collected on each player including stature using a portable stadiometer (Seca
213, Seca GMBH, Hamburg, Germany) and body mass using electronic scales (BWB-600, Tanita Corporation, Tokyo, Japan). For all games, players were fitted with microsensor units (OptimEye s5, Catapult Innovations, Melbourne, Australia) mounted at the upper torso, between the scapulae, in neoprene vests (Catapult Innovations, Melbourne, Australia). To reduce any potential between-device variability, players wore the same microsensor unit for each game across the season (Fox et al., 2019). External demands were measured via the $100-\mathrm{Hz}$ accelerometer, housed within the microsensor unit, and exported as raw instantaneous PlayerLoad ${ }^{\mathrm{TM}}$ (PL) via proprietary software (OpenField version 8, Catapult Innovations, Melbourne, Australia). PL is the proprietary metric of the microsensor, which represents the square root of the change in acceleration across the transverse (x), coronal (y), and sagittal (z) planes (Montgomery et al., 2010). The reliability of PL has been previously supported in team sport athletes (Luteberget et al., 2017).

Raw PL data were then exported and processed in RStudio (version 3.5.3) using the "zoo" package. Moving averages were calculated for PL across consecutive samples spanning $15 \mathrm{~s}$, $30 \mathrm{~s}, 1 \mathrm{~min}, 2 \mathrm{~min}, 3 \mathrm{~min}, 4 \mathrm{~min}$, and $5 \mathrm{~min}$. For each game, the highest intensity obtained by each player in each quarter for each sample duration was determined. Peak intensity was expressed as PL. $\mathrm{min}^{-1}$ by determining accumulated PL (sum of the raw PL across each duration), divided by 100, to represent the typical scaling factor applied (Montgomery et al., 2010). For each sample duration, PL was then reported relative to 1 min (e.g., the 15-s sample duration was multiplied by 4 to convert to PL. $\mathrm{min}^{-1}$, and the $5 \mathrm{~min}$ sample duration was divided by 5 to convert to PL·min ${ }^{-1}$ (Fox et al., 2020)).

Peak PL. $\mathrm{min}^{-1}$ in each quarter for each sample duration is reported as mean \pm standard deviation (SD). The normality of data distribution and sphericity were confirmed using the Shapiro-Wilk statistic and Levene's Test for equality of variances. For each sample duration, peak intensities in each game quarter were compared using linear mixed models with Bonferroni post hoc tests. The game quarter was entered as the fixed factor (4 levels), while the player $(n=8)$ was entered as the random term (Peugh, 2010). Effect sizes (Cohen's d) with 95\% confidence intervals were computed for all pairwise comparisons to identify the magnitude of differences between game quarters. Magnitudes were interpreted as trivial: $>0.20$, small: $0.20-0.59$, moderate: $0.60-1.19$, large: $1.20-1.99$, and very large: $\geq 2.00$ (Hopkins, 2006). Where confidence intervals for effect sizes crossed \pm 0.2 , the effect was interpreted as unclear (Hopkins et al., 2009). Linear mixed models and post-hoc tests were conducted using SPSS (Version 26, IBM Corporation, Armonk, USA) while effect sizes and confidence intervals were calculated using a customised Microsoft Excel spreadsheet (Version 15, Microsoft Corporation, Redmond, USA). Statistical significance was accepted where $\mathrm{p}<0.05$.

\section{Results}

Peak PL. $\mathrm{min}^{-1}$ across game quarters for each sample duration are presented in Figure 1. Pairwise comparisons in peak PL. $\mathrm{min}^{-1}$ between quarters for each sample duration are presented in Table 1. For the 15-s, 1-min, 2-min, 4-min, and 5 -min sample durations, differences in peak PL. $\mathrm{min}^{-1}$ between game quarters were non-significant, and effect sizes were trivial-small in magnitude $(\mathrm{p}>0.05)$. For the 30 -s sample duration, differences in peak PL. $\mathrm{min}^{-1}$ between game quarters were 


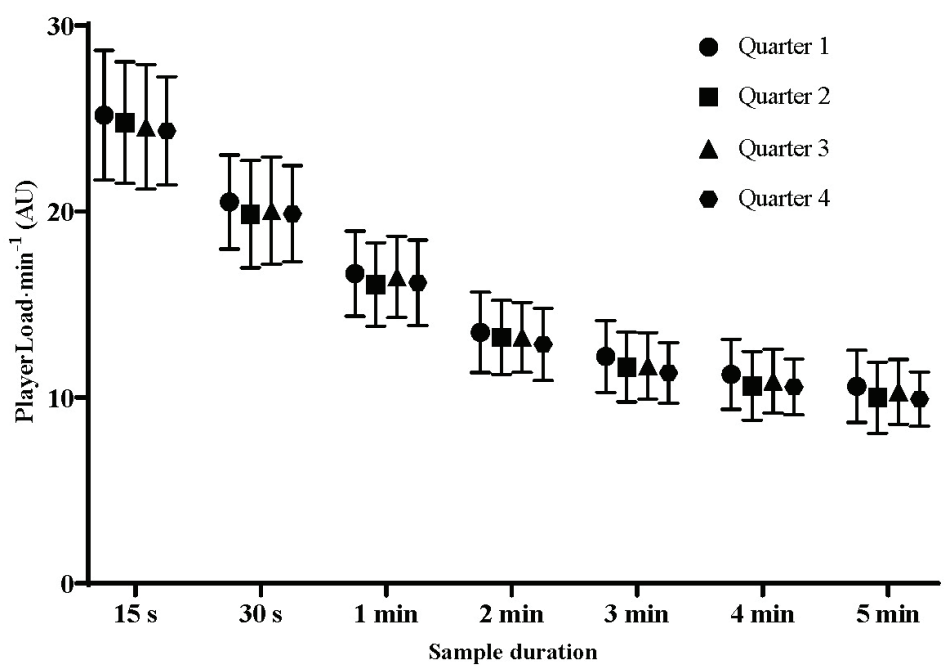

FIGURE 1. Peak intensity across basketball game quarters

non-significant, and effect sizes were unclear-small in magnitude $(\mathrm{p}>0.05)$. For the 3-min sample duration, there was a significant decline in peak PL. $\mathrm{min}^{-1}$ between Quarter 1 and
Quarter $4(\mathrm{p}=0.007$, small), with all other differences in peak $\mathrm{PL} \cdot \mathrm{min}^{-1}$ between quarters being non-significant and trivial-small in magnitude $(\mathrm{p}>0.05)$.

Table 1. Pairwise comparisons in peak PlayerLoad per minute between game quarters for each sample duration in semiprofessional, male basketball players.

\begin{tabular}{|c|c|c|c|}
\hline Sample duration comparisons & Effect size & $95 \% \mathrm{Cl}$ & $P$ \\
\hline \multicolumn{4}{|l|}{ 15-s sample } \\
\hline Quarter 1 vs Quarter 2 & 0.12 & $-0.12,0.36$ & 1.0 \\
\hline Quarter 1 vs Quarter 3 & 0.18 & $-0.06,0.43$ & 1.0 \\
\hline Quarter 1 vs Quarter 4 & $0.26^{*}$ & $0.01,0.51$ & 0.52 \\
\hline Quarter 2 vs Quarter 3 & 0.07 & $-0.18,0.32$ & 1.0 \\
\hline Quarter 2 vs Quarter 4 & 0.14 & $-0.11,0.39$ & 1.0 \\
\hline Quarter 3 vs Quarter 4 & 0.07 & $-0.19,0.32$ & 1.0 \\
\hline \multicolumn{4}{|l|}{ 30-s sample } \\
\hline Quarter 1 vs Quarter 2 & $0.24^{*}$ & $0.01,0.48$ & 0.56 \\
\hline Quarter 1 vs Quarter 3 & 0.17 & $-0.07,0.42$ & 1.0 \\
\hline Quarter 1 vs Quarter 4 & $0.25^{*}$ & $0.01,0.49$ & 0.73 \\
\hline Quarter 2 vs Quarter 3 & -0.07 & $-0.31,0.18$ & 1.0 \\
\hline Quarter 2 vs Quarter 4 & -0.01 & $-0.26,0.24$ & 1.0 \\
\hline Quarter 3 vs Quarter 4 & 0.06 & $-0.20,0.31$ & 1.0 \\
\hline \multicolumn{4}{|l|}{ 1-min sample } \\
\hline Quarter 1 vs Quarter 2 & $0.26^{*}$ & $0.02,0.50$ & 0.44 \\
\hline Quarter 1 vs Quarter 3 & 0.08 & $-0.16,0.32$ & 1.0 \\
\hline Quarter 1 vs Quarter 4 & $0.21^{*}$ & $-0.03,0.46$ & 0.90 \\
\hline Quarter 2 vs Quarter 3 & -0.19 & $-0.43,0.06$ & 1.0 \\
\hline Quarter 2 vs Quarter 4 & -0.04 & $-0.29,0.21$ & 1.0 \\
\hline Quarter 3 vs Quarter 4 & 0.14 & $-0.12,0.39$ & 1.0 \\
\hline \multicolumn{4}{|l|}{ 2-min sample } \\
\hline Quarter 1 vs Quarter 2 & 0.13 & $-0.11,0.37$ & 1.0 \\
\hline Quarter 1 vs Quarter 3 & 0.13 & $-0.11,0.37$ & 1.0 \\
\hline Quarter 1 vs Quarter 4 & $0.31^{*}$ & $0.07,0.56$ & 0.19 \\
\hline Quarter 2 vs Quarter 3 & 0.01 & $-0.25,0.25$ & 1.0 \\
\hline Quarter 2 vs Quarter 4 & 0.19 & $-0.06,0.44$ & 1.0 \\
\hline Quarter 3 vs Quarter 4 & $0.20^{*}$ & $-0.06,0.45$ & 1.0 \\
\hline
\end{tabular}




\begin{tabular}{|c|c|c|c|}
\hline Sample duration comparisons & Effect size & $95 \% \mathrm{Cl}$ & $P$ \\
\hline \multicolumn{4}{|l|}{ 3-min sample } \\
\hline Quarter 1 vs Quarter 2 & $0.30^{*}$ & $0.03,0.54$ & 0.18 \\
\hline Quarter 1 vs Quarter 3 & $0.27^{*}$ & $0.02,0.51$ & 0.37 \\
\hline Quarter 1 vs Quarter 4 & $0.49^{*}$ & $0.24,0.74$ & 0.007 \\
\hline Quarter 2 vs Quarter 3 & -0.04 & $-0.28,0.21$ & 1.0 \\
\hline Quarter 2 vs Quarter 4 & 0.18 & $-0.07,0.43$ & 1.0 \\
\hline Quarter 3 vs Quarter 4 & $0.22^{*}$ & $-0.03,0.48$ & 1.0 \\
\hline \multicolumn{4}{|l|}{ 4-min sample } \\
\hline Quarter 1 vs Quarter 2 & $0.33^{*}$ & $0.09,0.57$ & 0.095 \\
\hline Quarter 1 vs Quarter 3 & $0.20^{*}$ & $-0.04,0.44$ & 1.0 \\
\hline Quarter 1 vs Quarter 4 & $0.40^{*}$ & $0.15,0.64$ & 0.057 \\
\hline Quarter 2 vs Quarter 3 & -0.15 & $-0.39,0.10$ & 1.0 \\
\hline Quarter 2 vs Quarter 4 & 0.04 & $-0.21,0.29$ & 1.0 \\
\hline Quarter 3 vs Quarter 4 & $0.20^{*}$ & $-0.06,0.45$ & 1.0 \\
\hline \multicolumn{4}{|l|}{ 5-min sample } \\
\hline Quarter 1 vs Quarter 2 & $0.31^{*}$ & $0.07,0.55$ & 0.134 \\
\hline Quarter 1 vs Quarter 3 & 0.16 & $-0.09,0.40$ & 1.0 \\
\hline Quarter 1 vs Quarter 4 & $0.39^{*}$ & $0.14,0.64$ & 0.068 \\
\hline Quarter 2 vs Quarter 3 & -0.04 & $-0.29,0.20$ & 1.0 \\
\hline Quarter 2 vs Quarter 4 & 0.05 & $-0.20,0.30$ & 1.0 \\
\hline Quarter 3 vs Quarter 4 & $0.24^{*}$ & $-0.01,0.50$ & 0.944 \\
\hline
\end{tabular}

Note. $\mathrm{Cl}=$ Confidence Interval, Bolded $\mathrm{P}$ value indicates significant $(\mathrm{P}<0.05)$ difference, ${ }^{*}$ Indicates small effect size $(0.20-0.59)$.

\section{Discussion}

The present study is the first to compare peak external intensities encountered across game quarters in semi-professional basketball. Our data revealed that for all sample durations assessed, there was a small decrease in peak intensity encountered between the first and fourth quarters. In addition, for all sample durations, except $15 \mathrm{~s}$ and $2 \mathrm{~min}$, small declines in peak intensities were apparent between the first and second quarters. Our data also revealed small declines in peak intensity between the third and fourth quarters (2-, 3-, 4-, and 5-min sample durations) and first and third quarters (3- and 4-min sample durations).

In combination, our findings suggest that decreases in peak external intensities are evident across basketball games, with differences most prominent between the first and fourth quarters given this trend was revealed for all sample durations. Our findings also suggest that over longer sample durations ( $\geq 3 \mathrm{~min}$ ), peak intensity decreases from the first to second and third to fourth quarters. Differences in peak PL-min-1 across games may be related to fatigue-related mechanisms with past research suggesting that factors such as glycogen depletion and muscle damage contribute to decreases in external demands across basketball games (Scanlan et al., 2015). These fatigue-related mechanisms may also explain why small differences in peak external intensities across all sample durations were obtained between the first and fourth quarters, whereas small differences in peak external intensity were obtained between the first and second quarters and between the third and fourth quarters only over longer sample durations. Given that exercise intensity is mediated by duration, player's maximal effort likely cannot be maintained at the same intensity for extended periods, which explains why small decreases in intensity were apparent within the same game half, over longer sample durations. In this regard, the break between halves allows for greater recovery opportunity (15 min) compared to between quarters ( $2 \mathrm{~min}$ ), likely explaining the lack of any clear differences in peak $\mathrm{PL} \cdot \mathrm{min}^{-1}$ between the second and third quarters. In addition, longer sample durations likely include periods of inactivity or low-intensity activity (e.g., substitutions, time-outs, and stoppages in play for a change in possession of free-throw) which will also contribute to the lower intensities achieved. Lastly, the decline in peak intensities across games may also be related to tactical strategies, whereby game pace is reduced in later quarters to gain more ball control when in possession to increase the likelihood of successful game outcomes (Abdelkrim et al., 2007).

In interpreting the findings of the present study, some notable limitations should be considered. Data were collected on a semi-professional, male basketball team, so it cannot be assumed that the peak external intensities and differences in intensities observed between quarters are representative of female players (Scanlan et al., 2012) or players participating in other leagues or at other playing levels (Scanlan et al., 2011), suggesting that future work is needed to establish peak intensities encountered across various playing levels. In addition, only a single measure of intensity was assessed due to the frequent use of PL in basketball; however, when assessing game demands to optimize training prescription, other measures of intensity should also be explored.

Data from the present study suggest that peak external intensities decline across basketball games, with the most notable declines in intensity occurring between the first and fourth quarters. In addition, over longer sample durations $(\geq$ 
$3 \mathrm{~min}$ ) peak external intensity decreased within each half (i.e., between Quarters 1 and 2 and between Quarters 3 and 4). Therefore, basketball practitioners should assess not only total external demands or average external intensity across game quarters, but should also consider the most intense periods of activity encountered across different sample durations to assist in guiding training prescription. In this regard, using reference peak external intensity values from the first quarter may be useful, given these data represent the highest external intensities reached across the entire game. Specifically, preparing players to be able to maintain external intensities encountered in the first quarter during later game periods may assist in managing player fatigue and promoting optimal preparation for games. In further optimizing training prescription, attention should also be given to data captured over sample durations $\geq 3 \mathrm{~min}$ as these longer durations appear to provide further insights regarding fluctuations in peak external demands encountered within each game half.

\section{References}

Abdelkrim, N. B., El Fazaa, S., \& El Ati, J. (2007). Timemotion analysis and physiological data of elite under-19year-old basketball players during competition. British Journal of Sports Medicine, 41(2), 69-75. doi: 10.1136/ bjsm.2006.032318

Cunningham, D. J., Shearer, D. A., Carter, N., Drawer, S., Pollard, B., Bennett, M., Eager, R., Cook, C. J., Farrell, J., Russell, M., \& Kilduff, L. P. (2018). Assessing worst case scenarios in movement demands derived from global positioning systems during international rugby union matches: Rolling averages versus fixed length epochs. PLoS One, 13(4). doi: 10.1371/journal.pone.0195197

Fox, J., Conte, D., Stanton, R., McLean, B., \& Scanlan, A. (2020). The application of accelerometer-derived moving averages to quantify peak demands in basketball: A comparison of sample duration, playing role, and session type. Journal of Strength and Conditioning Research. In press. doi: 10.1519/ JSC.0000000000003486

Fox, J., O'Grady, C. J., \& Scanlan, A. T. (2019). The relationships between external and internal workload during basketball training and competition. International journal of sports physiology and performance. In press.

Garcia, F., Vázquez-Guerrero, J., Castellano, J., Casals, M., \& Schelling, X. (2020). Differences in physical demands between game quarters and playing positions on professional basketball players during official competition. Journal of Sports Science \& Medicine, 19, 256-263.

Hopkins, W. A. (2006). A scale of magnitudes for Effect Statistics. SportSci. Retrieved from http://www.sportsci. org/resource/stats/index.html

Hopkins, W., Marshall, S., Batterham, A., \& Hanin, J. (2009). Progressive statistics for studies in sports medicine and exercise science. Medicine and science in sports and exercise, 41(1), 3-12. doi: 10.1249/MSS.0b013e31818cb278

Impellizzeri, F. M., Marcora, S. M., \& Coutts, A. J. (2019). Internal and external training load: 15 years on. International Journal of Sports Physiology and Performance, 14(2), 270-273. doi: 10.1123/ijspp.2018-0935

Luteberget, L. S., Holme, B. R., \& Spencer, M. (2017). Reliability of wearable inertial measurement units to measure physical activity in team handball. International Journal of Sports Physiology and Performance, 13(4), 467473. doi: 10.1123/ijspp.2017-0036

Montgomery, P. G., Pyne, D. B., \& Minahan, C. L. (2010). The physical and physiological demands of basketball training and competition. International Journal of Sports Physiology and Performance, 5(1), 75-86. doi: 10.1123/ijspp.5.1.75

Peugh, J. L. (2010). A practical guide to multilevel modeling. Journal of School Psychology, 48(1), 85-112. doi: 10.1016/j. jsp.2009.09.002

Salazar, H., \& Castellano, J. (2019). Most demanding passages in Basketball: A preliminary study. Sport Performance \& Science Reports, 70(1). Retrieved from: https:// sportperfsci.com/wp-content/uploads/2019/08/SPSR73_ Salazar_190807_final.pdf

Scanlan, A. T., Dascombe, B. J., Reaburn, P., \& Dalbo, V. J. (2012). The physiological and activity demands experienced by Australian female basketball players during competition. Journal of Science and Medicine in Sport, 15(4), 341-347. doi: 10.1016/j.jsams.2011.12.008

Scanlan, A. T., Stanton, R., Sargent, C., O'Grady, C., Lastella, M., \& Fox, J. L. (2019). Working overtime: The effects of overtime periods on game demands in basketball players. International Journal of Sports Physiology and Performance, 14(10), 1331-1337. doi: 10.1123/ijspp.2018-0906

Scanlan, A. T., Tucker, P. S., Dascombe, B. J., Berkelmans, D. M., Hiskens, M. I., \& Dalbo, V. J. (2015). Fluctuations in activity demands across game quarters in professional and semi-professional male basketball. 29(11), 3006-3015. doi: 10.1519/JSC.0000000000000967

Scanlan, A., Dascombe, B., \& Reaburn, P. (2011). A comparison of the activity demands of elite and sub-elite Australian men's basketball competition. Journal of sports sciences, 29(11), 1153-1160. doi: 10.1080/02640414.2011.582509

Stojanović, E., Stojiliković, N., Scanlan, A. T., Dalbo, V. J., Berkelmans, D. M., \& Milanović, Z. (2018). The activity demands and physiological responses encountered during basketball match-play: A systematic review. Sports Medicine, 48(1), 111-135. doi: 10.1007/s40279-017-0794-z

Taylor, J. B., Wright, A. A., Dischiavi, S. L., Townsend, M. A., \& Marmon, A. R. (2017). Activity demands during multi-directional team sports: A systematic review. Sports Medicine, 47(12), 2533-2551. doi: 10.1007/s40279-0170772-5 